\title{
Public Abstract
}

First Name: Mevin

Middle Name: Brice

Last Name: Hooten

Degree: $\mathrm{PhD}$

Department: Statistics

Advisor's First Name: Christopher

Advisor's Last Name: Wikle

Graduation Term: Summer

Graduation Year: 2006

Title: Hierarchical Spatio-Temporal Model for Ecological Processes

Ecosystems are composed of phenomena that propagate in time and space. Often, ecological processes underlying such phenomena are studied separably in various subdisciplines, while larger scale, interlinking mechanisms are overlooked or only speculated about. As grows the burden of global climate change and human disturbance of natural systems, so grows the need for rigorous statistical methods focused on characterizing and forecasting large-scale spatio-temporal environmental and ecological processes in the presence of limited data and multiple sources of uncertainty.

Hierarchical models offer a powerful means with which to study complex phenomena in space and time. This dissertation develops and illustrates the utility of spatio-temporal hierarchical models for studying ecological phenomena. 